\title{
\begin{tabular}{l|l} 
pcori)? & PATIENT-CENTERED OUTCOMES RESEARCH INSTITUTE \\
RESEARCH SUMMARY
\end{tabular}
}

\section{Using Visual Analytic Methods to Identify Patient Groups}

Principal investigator

Suresh K. Bhavnani, PhD
Organization

The University of Texas Medical Branch at Galveston

\section{What was the project about?}

Patients with multiple health problems, such as diabetes and heart disease, may benefit from personalized treatment approaches. For example, patients who have both breathing problems and diabetes may need different medicines than patients who have diabetes alone. Researchers can use statistical methods to group patients with specific health problems and figure out how well treatments work for those patients. But current methods don't always find all the health problems that can affect how treatments work.

In this project, the research team developed a new method to group patients with common health problems. The method, called a visual analytic method, used a computer program and patient data to draw pictures or maps of the patient groups. The method helped the research team figure out the chance of returning to the hospital for patients in each group.

\section{What did the research team do?}

The research team used Medicare claims data for patients with COPD, a lung problem that makes it hard to breathe. The team looked at data for 29,016 patients who returned to the hospital within 30 days of discharge. Then they looked at data for 29,016 patients who didn't return to the hospital within 90 days of discharge. The team matched patients who did and didn't return to the hospital based on their age, gender, race, and income.

The research team then used the new method to identify and group patients who returned to the hospital, based on their health problems. The method created a picture showing how the groups were similar and different. Then the team used statistical methods to figure out the chance of returning to the hospital for patients in each group. The method also showed a patient's likelihood of being in a specific group.

Doctors helped the research team develop the methods and review the results.

\section{What were the results?}

The method displayed a picture of four groups of patients with COPD based on their most common health problems:

- Patients with high blood pressure

- Patients with diabetes with complications, kidney failure, and heart failure

- Patients with mental health problems and social concerns

- Patients with organ damage and digestive conditions

The method also identified the chance of returning to the hospital for each group. For example, the patients with diabetes group had an 18 percent chance of returning to the hospital.

\section{What were the limits of the project?}

The new method took almost a week for a computer to run. The research team used claims data. Results 
may have differed if the team used other types of data or selected patients in different ways.

Future studies could test these methods using other types of data such as health records.

\section{How can people use the results?}

Researchers could use these methods to identify groups of patients with certain health conditions, and figure out how treatments work for patients in those groups.

To learn more about this project, visit www.pcori.org/Bhavnani360. 